\title{
Simulation of Cracking in High Concrete Gravity Dam Using the Extended Finite Elements by ABAQUS
}

\author{
Albaadani Ghallab \\ Department of Engineering Mechanics, Hohai University, Nanjing, China \\ Email address: \\ ghallab@hhu.edu.cn \\ To cite this article: \\ Albaadani Ghallab. Simulation of Cracking in High Concrete Gravity Dam Using the Extended Finite Elements by ABAQUS. American \\ Journal of Mechanics and Applications. Vol. 8, No. 1, 2020, pp. 47-55. doi: 10.11648/j.ajma.20200801.12
}

Received: December 8, 2019; Accepted: December 30, 2019; Published: January 8, 2020

\begin{abstract}
China's energy demand is growing rapidly and large hydropower plants are becoming more common. This therefore has resulted in the building of many large concrete dams across the country. Significant dam cracking can have major environmental, economic and social consequences. Collapse of High concrete dam may cause serious harm to economic development as well as safety of the people; thereby necessitating the need for research on safety and stability of high concrete dam under high water and seismic conditions. In this paper, the high concrete dam of Longtan was used as an example, using the ABAQUS program based on the extended finite element method to analyze the Longtan Dam under static and dynamic conditions which has been commonly used for the study of crack growth. A numerical prediction of crack propagation in concrete gravity dams is presented. The two-dimensional seismic numerical study was done using acceleration-time records from Koyna earthquake in 1976 Dam concrete cracking range and cracking were discussed during a seismic time. The locations that are easy to generate cracks are illustrated from the analysis and the findings showed that, during seismic activity the Longtan Dam was rather unsafe. The results of the work can provide a valuable guide for the design and operation of the dam system.
\end{abstract}

Keywords: Gravity Dam, Crack, XFEM, Abaqus

\section{Introduction}

The development of society in China is proliferating; as a result of this, the demand for energy is growing. Today in China, coal burning is the most common source of energy production, an area that continues to expand. Coal-burning, however, has disadvantages such as high carbon dioxide $\left(\mathrm{CO}_{2}\right)$ emissions, pollution, and increased mercury concentration in rivers. To face China's energy needs, one solution is to improve its second-largest source of energy, hydropower, which currently provides 15 percent of total energy production in the country. It is worth noting that China is the largest hydropower producer in the world; also, there are plans to build 13 large hydropower plants, some of which are already under construction. Longtan Dam, a concrete dam of high density, is one of the dams in the country and has recently started producing electricity. Longtan Dam is the highest dense concrete dam in the world with a height of 216.5 meters and produces $6300 \mathrm{MW}$. Several risks arise from the construction and operation of the dam on a scale where a severe failure of the dam can lead to devastating consequences for the environment and society, which will lead to significant economic consequences. Therefore, safety and sustainability issues should be of the utmost importance from design and construction to maintenance and operation.

Many of China's dams are located in seismic high-risk areas, increasing the risk of dam destruction. Modeling structures to deal with stability-related issues can provide useful information and help enhance the safety of the dam and reduce building costs. Therefore, even if the probability of their occurrence is low, earthquakes serve as an important threat to research.

Concrete dam is weak in tension; concrete structures are prone to cracking. In recent decades, extensive studies have been carried out proceeding the behavior of concrete in failure. Kaplan [1] proposed the thesis of fracture in a concrete crack. The main destruction methods are theoretical analysis, experimental studies, numerical analysis. Numerical analysis is suitable for problems with arbitrary geometry and 
boundary conditions, so it has been used abroad in concrete fracture. Key technologies that need to be solved using an extended finite element method are a specific constitutive model and a crack model.

The extended method of finite elements (XFEM) [2, 3], based on the method of cohesive segments [4] in conjunction with the technique of phantom nodes $[5,6]$, Simulating the initiation and propagation of a crack along an arbitrary path can be used. Since the crack propagation is not tied to the mesh element boundaries. There is no need to determine the crack tip position with this approach, but we can determine the area of control in which the crack propagates. There is no need for asymptotic singularity at the near end, and only a displacement jump is required across the fractured element. Therefore, the crack must propagate at the same time throughout the component to avoid simulating the singularity of the stress. The method of the phantom node describes discontinuity by superimposing the phantom elements instead of introducing additional degrees of freedom, and it is easy to incorporate into conventional finite element codes. The XFEM applies to $2 \mathrm{D}[5,7,8]$ as well as dynamic issues $[6,9$, $10]$.

Pekau and Yuzhu [11] After earthquakes, the distinct Element Method (DEM) was used to study the seismic activity of the collapsed Koyna Dam. Their study showed that if the crack shape was horizontal or inclined upstream, dam safety was ensured, and if the crack leaned downstream, it was dangerous. Javanmardi [12] In order to study the seismic resistance of concrete gravity dams a discrete crack model was combined with a theoretical model to adjust the uplift pressure along a dam crack. Wang [13] A threedimensional discontinuous strain analysis (3D-DDA) was used to test the strength of the gravity dam on the articulated rock base and seismic resistance of the collapsed Konya gravity dam. Pekau and Zhu $[14,15]$ To research the seismic behavior of concrete dams with cracks, a rigid model and a flexible FE model are implemented. Mirzayee [16] proposed a hybrid approach using separate elements and boundary elements (DE-BE) to simulate the nonlinear seismic behavior of destroyed concrete gravity dams taking into account the effects of the interaction of the dam and the reservoir.

Amir Pirooznia [26] Considering obtained results revealed that the layer can have the reducing effect on responses and crack propagation process of dam model. Hamid Mohammadnezhad [25] the massed and massless foundation results are compared and it is concluded that the massless foundation approach leads to the overestimation of the displacements and stresses within the dam body.

Thus, if the dam fails in these areas, it contributes not only to direct economic losses but also to substantial indirect losses. During the desired period, the dam's seismic characteristics must be taken into account. In this paper, we study the effects of cracking under pressure, gravity and the seismic response of a concrete dam, using a numerical model based on the Longtan Dam in China. The findings may be a guide to the strength and safety of the dam.

\section{XFEM for Dynamic Analysis of Cracks}

Modeling stationary discontinuities, such as fractures, using the conventional method of finite elements requires the mesh to match geometric discontinuity. Therefore, in the area of the crack tip, significant mesh refinement is necessary for adequate capture of singular asymptotic fields. Modeling a spreading crack is even more difficult since the mesh needs to be constantly revised to match the structure of the fracture as the crack grows.

The extended finite element method (XFEM) removes the inconvenience associated with crack meshing engagement. The extended finite element method (XFEM) was first introduced [2]. This approach is an extension of the traditional method of finite elements based on the concept of unity partition [17], which makes it easy to include local enrichment functions in the finite element approximation. Special enriched functions ensure the presence of gaps in combination with degrees of additional freedom. The structure and properties of finite elements, such as sparseness and symmetry, are preserved.

\subsection{Approximation of XFEM}

XFEM enriches with discontinuous functions the standard displacement of finite elements. The approximation of the displacement vector function with the unit enrichment partition (Figure 1) The XFEM takes form [8, 18]:

$$
u_{x f e m}(x)=\sum_{i \in l} u_{i} N_{i}(x)+\underbrace{\sum_{j \in J} b_{j} N_{j}(x) H(x)}_{\text {only Heaviside nodes }}+\underbrace{\sum_{k \in K 1} N_{k}(x)\left(\sum_{l=1}^{4} c_{k}^{l 1} F_{l 1}(x)\right)+\sum_{k \in k_{2}} N_{k}(x)\left(\sum_{l=1}^{4} c_{k}^{l 2} F_{l 2}(x)\right)}_{\text {only crack-tip nodes }}
$$

Where $x=\{x, y\}$ Is a two-dimensional system of coordinates, I is the set of all mesh nodes, $N_{i}(x)$ is the shape function attached to the node $i, u_{i}$ Are the classic degrees of node freedom $i . \mathrm{J} \subset \mathrm{I}$ Is the group of nodes whose shape function is cut through a crack, $b_{j}$ is the corresponding vector of additional degrees of freedom to model crack faces (not crack tips). If the crack is aligned with the mesh, $b_{j}$ represents the crack opening, $H(x)$ is the Heaviside function. $K_{1} \subset I$ and $K_{2} \subset I$ A set of nodes whose shape function support contains, respectively, the first and second crack tips in their domain of influence. $c_{k}^{l 1}$ and $c_{k}^{l 2}$ are the corresponding vectors of additional degrees of freedom related to the modeling of crack tips, since the near-tip regions are enriched by four different crack 
functions. $F_{l 1}(x)$ and $F_{l 2}(x)$ are the role of crack-tip enrichment. If there is no enrichment, the equation above reduces the approximation of the classical finite element $u_{f e m}(x)=\sum_{i} u_{i} N_{i}(x)$ of The first term on the right side of the above formula refers to all nodes, while the second term applies to nodes whose shape function support is cut through the inside of the crack, and the third (fourth) term applies only to nodes where the shape function support is cut through the crack edge.

\subsection{Functions of Enrichment}

For crack analysis, usually, enrichment functions consist of near-tip asymptotic functions that capture the singularity surrounding the crack tip and a discontinuous function that defines the change in displacement between the crack surfaces. The estimation of the unit enrichment partition for a displacement vector function is

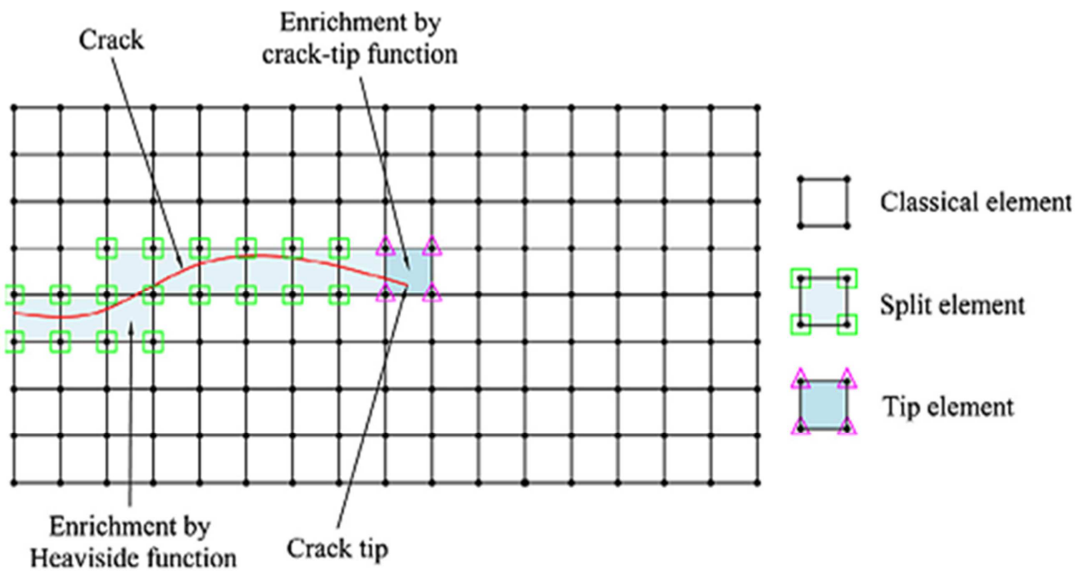

Figure 1. Enriched nodes in the XFEM. [19].

$$
u=\sum_{I=1}^{N} N_{I}(x)\left[u_{I}+H(x) a_{I}+\sum_{\alpha=1}^{4} F_{\alpha}(x) b_{I}^{\alpha}\right]
$$

where $N_{I}(x)$ are the usual shape functions of nodal, the first term of this equation, $u_{I}$, is the normal nodal displacement vector connected to the continuous part of the FE solution; the second term results from the enriched nodal degree of vector freedom; $a_{I}$, and the associated discontinuous jump function $H(x)$ The product of the nodal enriched degree of freedom vector is the third term on the crack surfaces, $b_{I}^{\alpha}$, and the associated elastic asymptotic crack-tip functions, $F_{\alpha}(x)$. The first term on the right side applies to all nodes in the model; the second term applies to nodes whose shape supports the function of crack interior cuts, and the third term applies only to nodes whose shape supports the function of crack tip cuts.

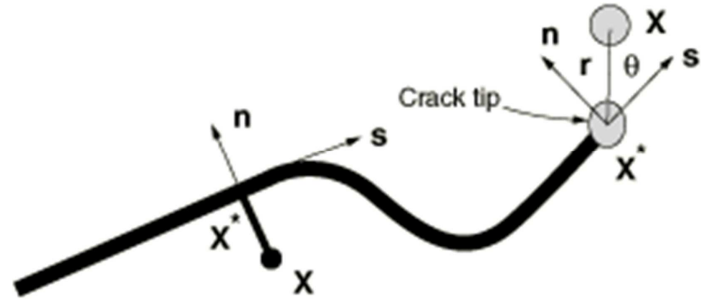

Figure 2. Illustration of normal and tangential coordinates for a crack. [20].

Figure 2 Illustrates the discontinuous jump function across the crack surfaces, $H(x)$, which is given by

$$
H(x)= \begin{cases}1 & \text { if }\left(x-x^{*}\right) \cdot n \geq 0 . \\ -1 \quad \text { otherwise, }\end{cases}
$$

where $x$ is a sample (Gauss) point, $x^{*}$ is the point nearest to the crack $x$, and $n$ Is the unit normal to the crack from the outside $x^{*}$.

Figure 2 Illustrates the functions of asymptotic crack tips in isotropic elastic material, $F_{\alpha}(x)$, which are given by

$$
F_{\alpha}(x)=\left[\sqrt{r} \sin \frac{\theta}{2}, \sqrt{r} \cos \frac{\theta}{2}, \sqrt{r} \sin \theta \sin \frac{\theta}{2}, \sqrt{r} \sin \theta \cos \frac{\theta}{2}\right]
$$

where $(r, \theta)$ is a polar coordinate system located at the tip of the crack and $\theta=0$ is tangent to the tip of the crack. These functions span elastostatics' asymptotic crack-tip function, and $\sqrt{r} \sin \frac{\theta}{2}$, Consider discontinuity along the surface of the crack. The use of asymptotic crack-tip functions in an isotropic elastic material is not limited to crack modeling. In addition to a bi-material interface, impinged on the bimaterial interface, or in an elastic-plastic power-law hardening material, the same approach can be used to represent a crack. However, different types of asymptotic crack-tip functions are needed in each of these three cases based on the crack area and degree of deformation of the inelastic material. Sukumar and Prevost and Elguedj discuss the various types of asymptotic crack-tip functions [19, 20, 21].

Accurately modeling the crack-tip singularity requires 
continuously keep following the crack propagation is difficult because the degree of crack singularity in a nonisotropic material based on the crack zone. Therefore, only when modeling stationary cracks in Abaqus / Standard, we presume the asymptotic singularity functions. One of the two methods mentioned below is used to model propagate cracks.

One alternative method within XFEM is based on the cohesive behavior of traction-separation. This approach is used for simulating crack propagation and initiation in Abaqus/Standard. This is a widespread ability to model interaction that can be used to model fracture that is brittle or ductile. The other Abaqus / Standard crack initiation and propagation capabilities are based on cohesive elements or surface-based cohesive behavior [20]. Unlike these methods, which require cohesive surfaces to follow boundaries of elements and cracks to propagate along a set of predefined paths, the method of XFEM-based cohesive segments can be used to simulate crack propagation and initiation in bulk materials along an arbitrary, solution-dependent path, as crack propagation is not attached to the mesh component boundaries. The near-tip asymptotic singularity is not required in this case, and only the displacement leap across a cracked element is taken into account. Therefore, to prevent the need to design the stress singularity, the crack must propagate across the whole element at a time.

Phantom nodes are added to the original real nodes to show the discontinuity of the cracked elements, as illustrated in Figure 3. Every phantom node is ultimately restricted to its similar real node when the element is undamaged. The cracked element divides into two parts when the element is divided by a crack. That part is made by combining some real and phantom nodes based on the crack's orientation. Each phantom node, together with its related real node, is no longer attached and can shift apart.

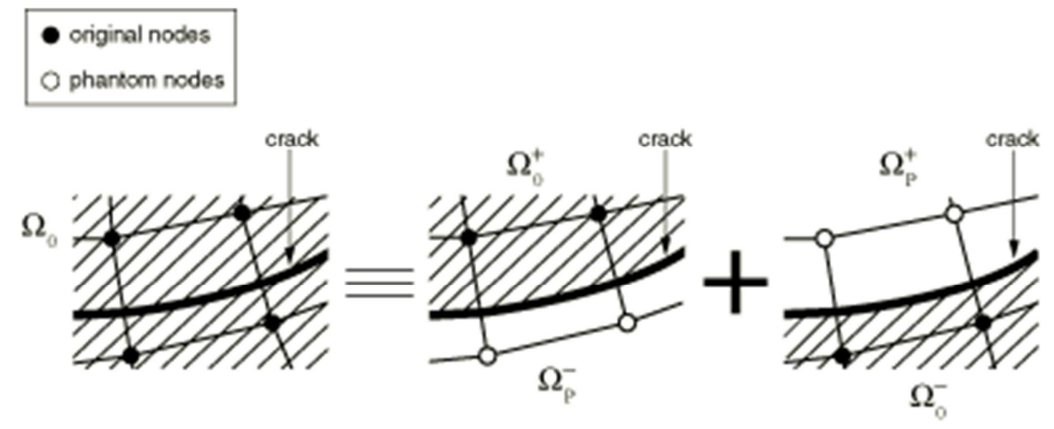

Figure 3. The principle of the phantom node method. [20].

The cohesive law dictates separation magnitude until the cohesive strength of the cracked element reaches zero, after which the phantom and the actual nodes pass separately. The part of the cracked element that belongs to the real domain to have a group of full interpolation bases, $\Omega_{0}$, Extends to the domain of phantoms, $\Omega_{p}$. Then the real domain displacement, $\Omega_{0}$, The degrees of freedom for nodes in the phantom domain can be interpolated, $\Omega_{p}$. The change in the displacement field is rendered by simply integrating from the side of the real nodes to the crack only over the area; i.e., $\Omega_{0}^{+}$and $\Omega_{0}^{-}$. This method provides an attractive and useful engineering approach and was used by Song and Remmers to simulate the initiation and growth of multiple cracks in solids $[4,6]$. If the mesh is sufficiently refined it has been verified to have almost no mesh dependence.

\section{Validation Test}

The numerical model was created to confirm the effectiveness of a two-dimensional model of a separate monolith was designed to investigate the Koyna concrete dam's crack resistance during the Koyna earthquake of 1967. The Koyna earthquake's two components are shown in Figure 5. Only the horizontal and vertical components of the earth's seismic movement are taken into account in the verification test, which is consistent with the seismic data reported in the model test [24].

The 2D model's geometric characteristics and FE mesh are shown in Figure 4. The material specifications for the Koyna dam are as follows. Concrete tensile and compressive strengths are respectively $2.9 \mathrm{MPa}$ and $24.1 \mathrm{MPa}$. The fracture energy is $250 \mathrm{~N} / \mathrm{m}$. The foundation rock is assumed to be massless in order to avoid wave propagation effects when adding free field earthquake records at the base. For the tensile strength, a dynamic magnification factor of 1.2 is assumed to compensate for the effects of the strain.

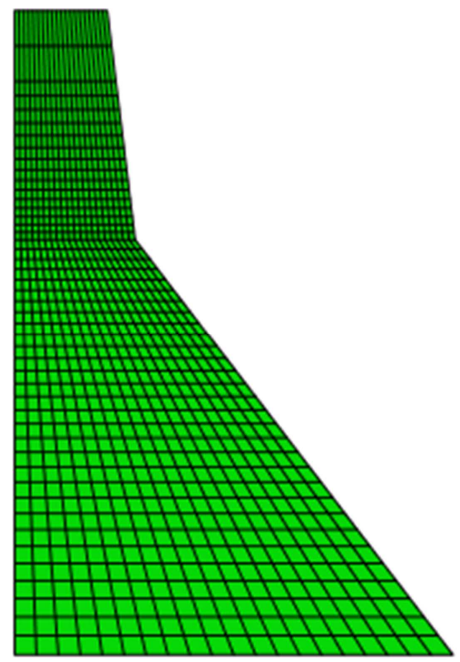

Figure 4. Koyna FEM model mesh. 


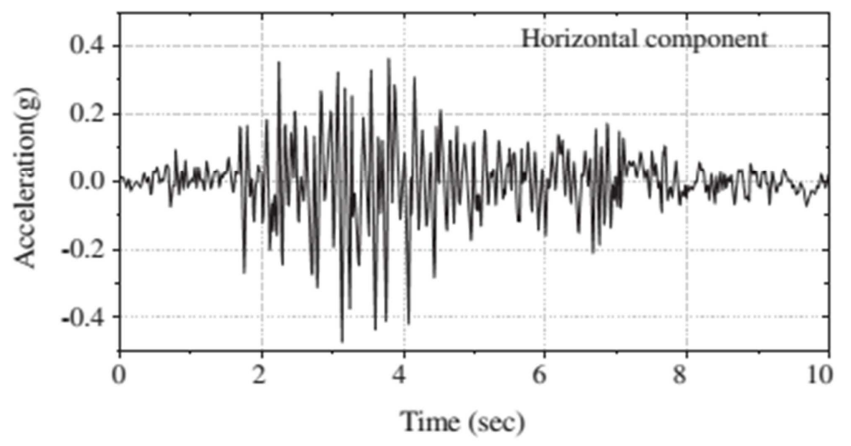

(a)

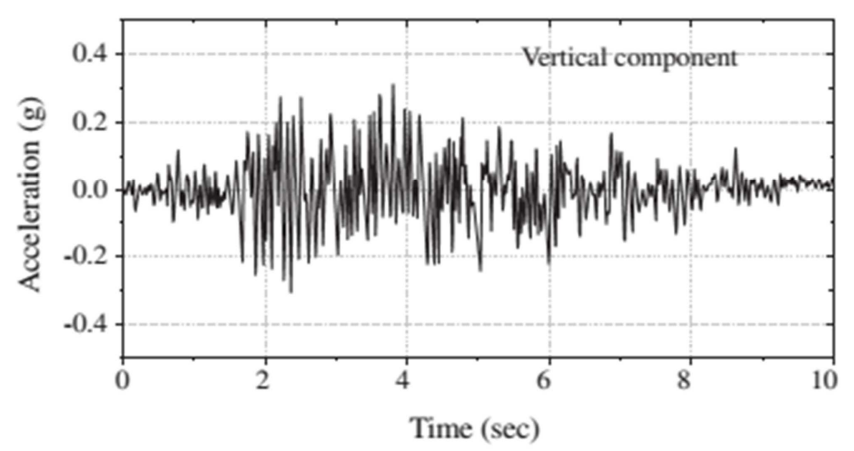

(b)

Figure 5. Koyna earthquake on December 11, 1967: (a) horizontal component and (b) vertical component.

Figure 6 show the displacement of the crest dam of koyna

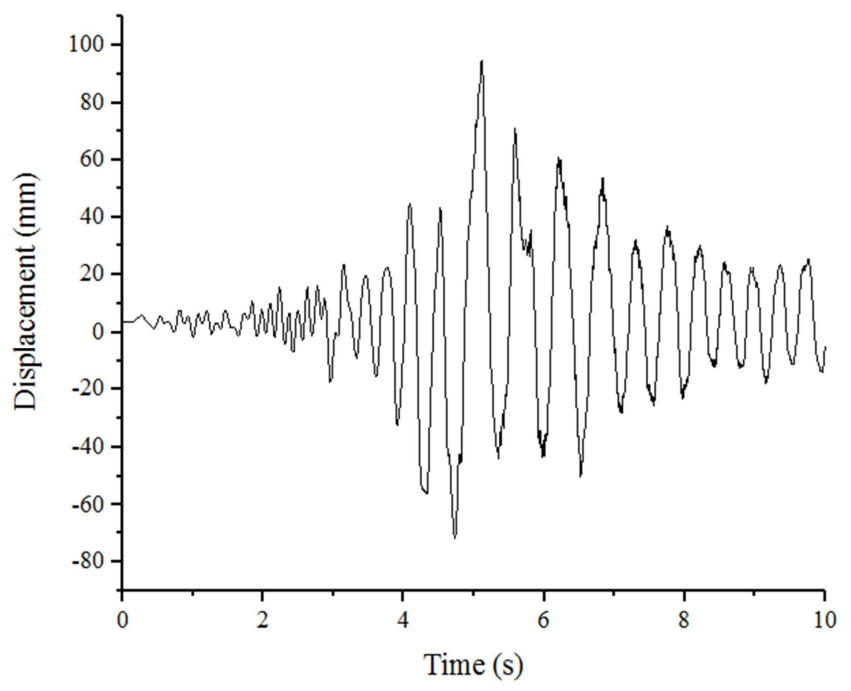

Figure 6. Displacement of the crest dam of koyna.

The nodal displacements at the truncated boundary of the reservoir and the foundation are assumed to be equal to zero in the normal direction for the initial time step. In addition, the foundation's lower boundary is completely limited. All movement restrictions are removed after a static analysis and the flow and vertical components of the Koyna earthquake accelerations are added as an input load to the base of the foundation. In Figure 7, Final Koyna dam crack profile obtained by numerical modeling with design test profile [24]. The damage area is shaded in green showing crack profiles.
The numerical cracking model, as can be seen clearly, fits quite well with the experimental results. We can conclude that the numerical scheme presented can effectively predict the crack propagation process under seismic loads in concrete gravity dams.
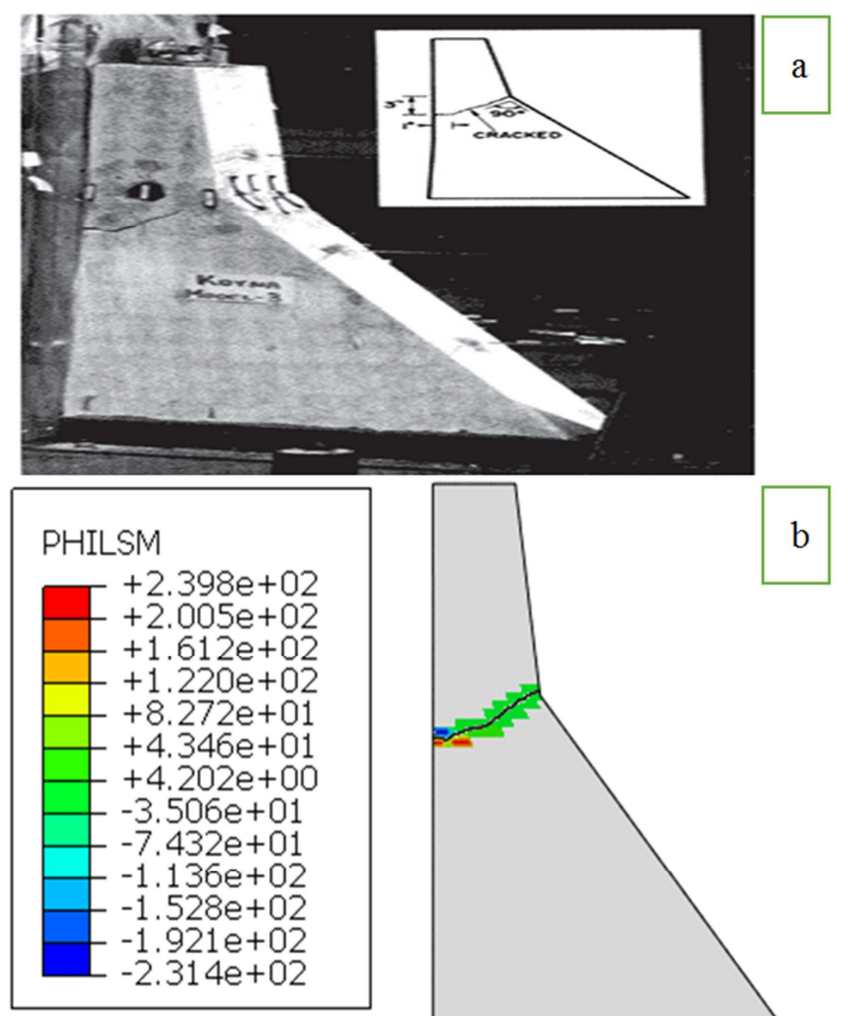

Figure 7. a) Crack experimental [24] and b) Crack path of Koyna dam.

\section{Longtan Gravity Dam Seismic Crack Analysis Based on XFEM}

The Longtan Gravity Dam in China, 216 meters high, is the highest concrete dam we will simulate the damage that can be caused under a similar earthquake in Koyna. The Koyna earthquake with a magnitude of 6.5 on the Richter scale, with maximum acceleration measured in the base gallery of $0.49 \mathrm{~g}$ and $0.34 \mathrm{~g}$ in the horizontal and vertical direction, caused severe damage to the structure of the dam, so we will show the seismic effect in a high dam.

In this paper, the Longtan dam's dynamic cracking analysis is carried out using the XFEM for the concrete material. The Koyna earthquake's history of time is shown in Figure 5. The finite element model for the highest dam is shown in Figure 8.

The material parameters are as follows for Longtan concrete dam: the elasticity modulus $E=1.97 \times 10^{4} \mathrm{MPa}$, the Poisson's ratio $v=0.167$, the mass density $\rho=2400 \mathrm{~kg} / \mathrm{m}^{3}$ The dam's tensile strength and compressive strength are respectively 1.57 and $18.5 \mathrm{MPa}$. the fracture energy is $255 \mathrm{~N} / \mathrm{m}$. For the tensile strength, a dynamic magnification factor of 1.2 is assumed to account for the 
effects of the strain rate. The Rayleigh damping system, with a damping ratio of $5 \%$, considers the energy distribution of the monolith. Consideration the maximum reservoir water level of $190 \mathrm{~m}$.

Analysis of dynamic crack propagation of the Longtan gravity dam under the Koyna earthquake is carried out using the system of cohesive segments based on XFEM made in ABAQUS. The time step of integration used in the analysis is $0.01 \mathrm{~s}$. It defines the response of cohesive behavior in the model's enriched elements. The maximum criterion for principal stress failure is selected for the initiation of damage.

Dam crest (point $\mathrm{N}$ ) obtained history of horizontal and vertical displacement using linear elasticity and XFEM is shown in Figure 9. The horizontal and vertical displacement positives are respectively in the downstream and upward directions. The cracking initiation began at (1.80s), which means that during the relatively small amplitude motion the maximum principal stress in the dam reaches the tensile strength of the concrete, and the cracking begins at $\mathrm{t}=1.8 \mathrm{~s}$.

From Figure 9, this is obvious That the propagation of the crack often changes the vibration response periods and an increased vibration period of displacement response from the XFEM method is observed, indicating that the rigidity of the dam is gradually reduced due to concrete softening.

Figure 10 shows the maximum principal stresses in the element $\mathrm{Z}$ at the downstream face slope. It is seen from Figure 11. After cracking the tensile strength is eliminated. For these situations, the average peak values of the maximum principal stresses are shown in the Figure 10 about $1.8 \mathrm{MPa}$.

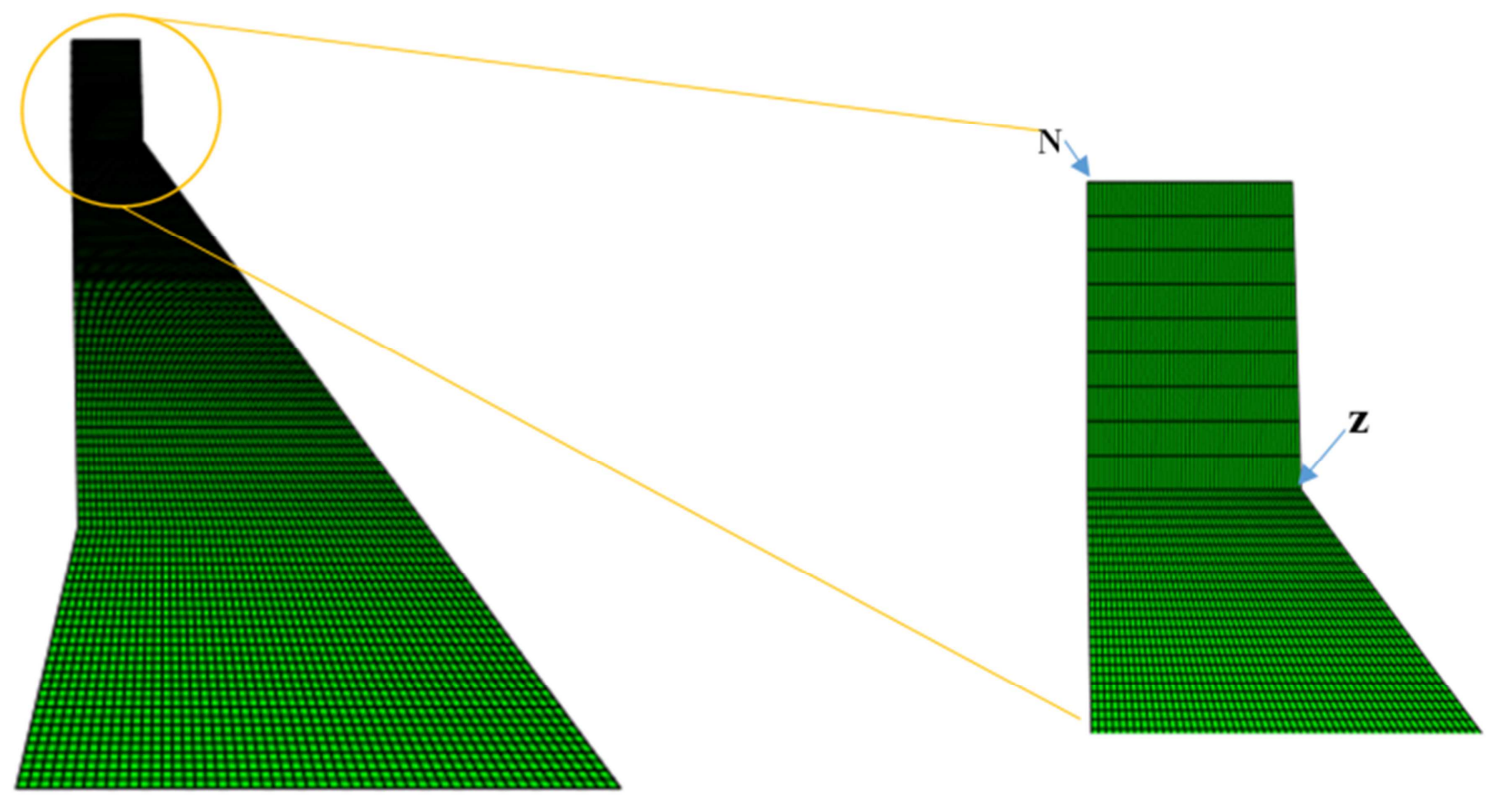

Figure 8. Longtan dam's finite element model.
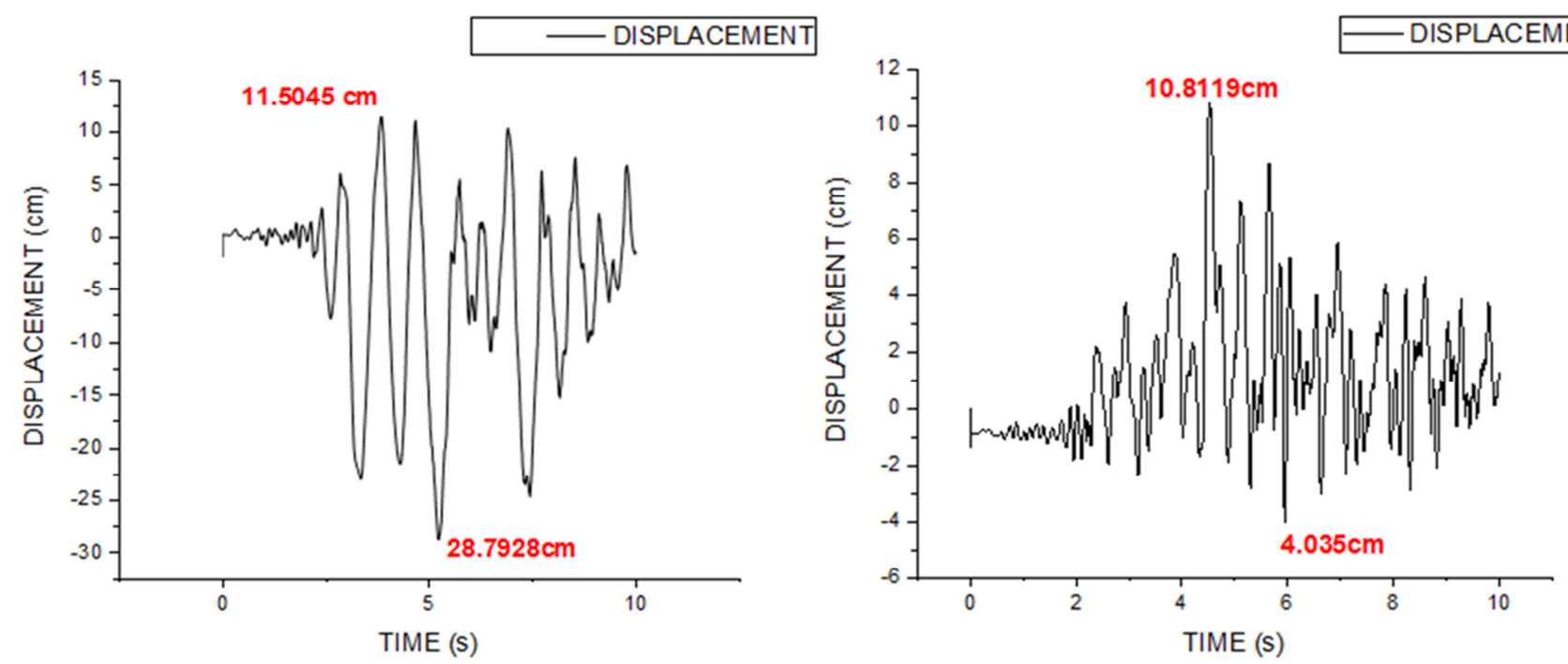

Figure 9. Horizontal and vertical displacements on the crest of the dam: (a) horizontal displacement and (b) vertical displacement. 


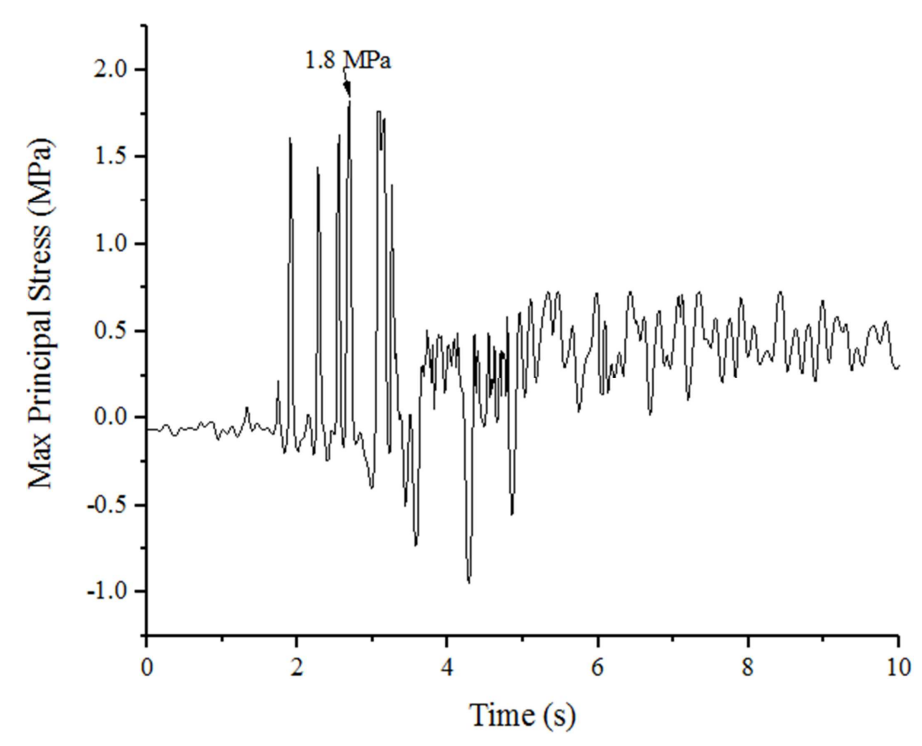

Figure 10. Graphs of the maximum principal stresses occurred in element $Z$.
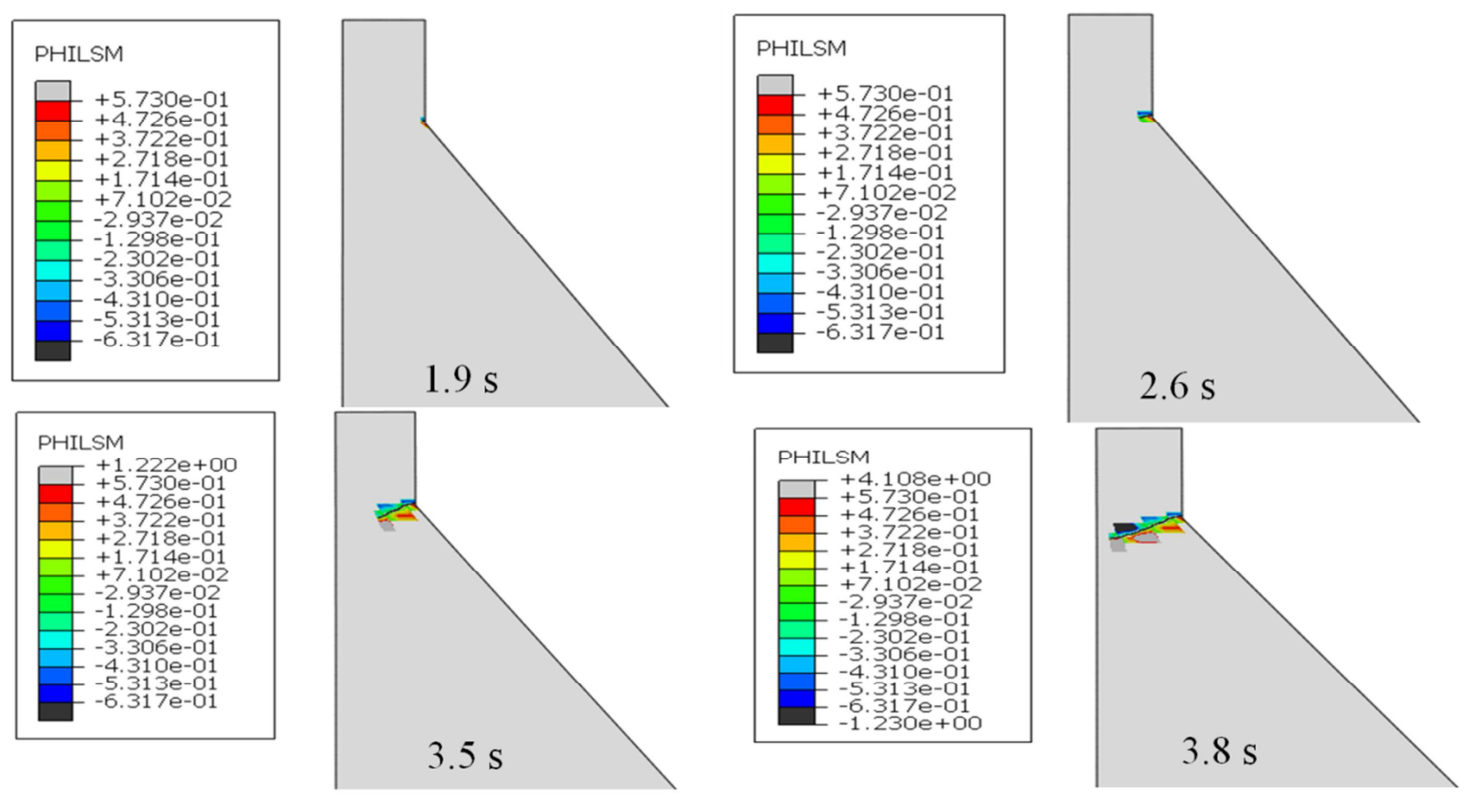

Figure 11. Longtan dam process propagation crack at four selected intervals. (a) Time $A(t=1.8 \mathrm{~s})$, (b) Time $B(t=2.6 \mathrm{~s})$, (c) Time $C(t=3.5 \mathrm{~s})$ and (d) Time $D$ $(t=3.8 s)$.

Figure 11 As shown, smooth curvature discrete cracks are obtained that penetrate the elements. The process of propagation fractures can be defined as follows: The initial crack in the dam is first detected at $1.9 \mathrm{~s}$ where the downstream face slope shifts. Figure 11 There is a concentration of stress at this location and high values are taken from the tensile stresses. The crack reaches deeper inside the dam as the vibration characteristics. After that, through the length of the dam segment, the downstream crack propagates about three-fourth. Due to the compressive stresses arising from top block rocking the crack path curves down. It can be noted that the crack propagation cycle is primarily concentrated from $1.9 \mathrm{~s}$ to $3.9 \mathrm{~s}$ at the time when the dam crest's dynamic displacement response is greater and the dam retains its overall protection.

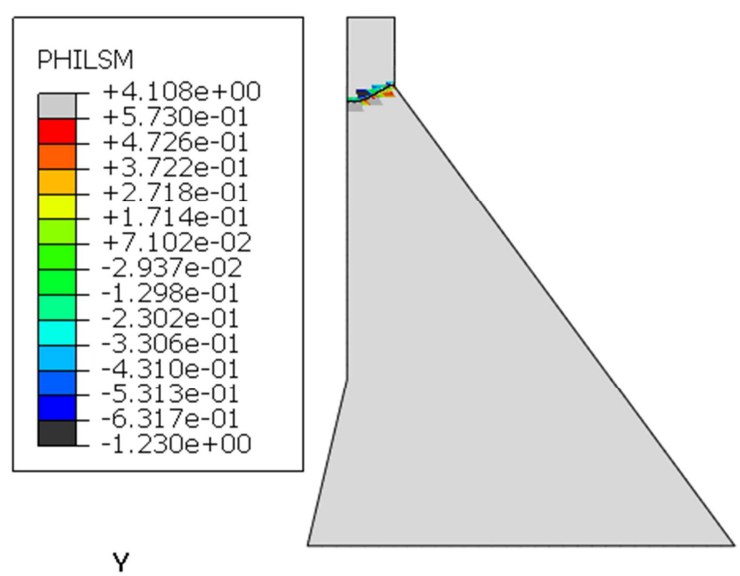

Figure 12. The final failure Profile of Longtan dam under the 1967 earthquake of koyna. 
Figure 12 shows Longtan Dam's final crack profile under the Koyna earthquake of 1967. It can be noted that the crack profile that exists matches the validation results of the Koyna dam model fairly well. It can be assumed that, under seismic conditions, the XFEM technique can effectively predict the crack propagation process in concrete gravity dams.

\section{Conclusions}

In terms of enduring an earthquake with an amplitude of $0.4 \mathrm{~m} / \mathrm{s}$, the Longtan Dam turns out to be rather unstable, and the crack occurs rapidly in a short time and amplitude which can depend on several factors that have already been pointed out. Earthquakes are uncommon in the region where the hydropower station is located, and this is likely the most critical factor behind the outcome, along with the very extreme case assumed in the simulations, i.e., the uplift force and the weak interface. This paper points out the most critical areas, but before providing any advice on the damage, further investigations need to be performed. A major failure of the dam could have serious effects on the environment, economy and society. Therefore, Research on dam protection should continue and be more improved to make the use of hydropower as safe as possible and to have as little impact as possible on the surrounding environment. study must also take into consideration the crucial advantages that hydropower brings to a developing country as China.

\section{References}

[1] M. F. Kaplan, "Crack propagation and the fracture of concrete," in Journal Proceedings, 1961, vol. 58, no. 11, pp. 591-610.

[2] T. Belytschko and T. Black, "Elastic crack growth in finite elements with minimal remeshing," Int. J. Numer. Methods Eng., vol. 45, no. 5, pp. 601-620, 1999.

[3] F. L. Stazi, E. Budyn, J. Chessa, and T. Belytschko, "An extended finite element method with higher-order elements for curved cracks," Comput. Mech., vol. 31, no. 1-2, pp. 38-48, 2003.

[4] J. J. C. Remmers, R. de Borst, and A. Needleman, "The simulation of dynamic crack propagation using the cohesive segments method," J. Mech. Phys. Solids, vol. 56, no. 1, pp. 70-92, 2008.

[5] A. Hansbo and P. Hansbo, "A finite element method for the simulation of strong and weak discontinuities in solid mechanics," Comput. Methods Appl. Mech. Eng., vol. 193, no. 33-35, pp. 3523-3540, 2004.

[6] J. Song, P. M. A. Areias, and T. Belytschko, "A method for dynamic crack and shear band propagation with phantom nodes," Int. J. Numer. Methods Eng., vol. 67, no. 6, pp. 868893, 2006.

[7] N. Moës and T. Belytschko, "Extended finite element method for cohesive crack growth," Eng. Fract. Mech., vol. 69, no. 7, pp. 813-833, 2002.
[8] N. Moës, J. Dolbow, and T. Belytschko, "A finite element method for crack growth without remeshing," Int. J. Numer. Methods Eng., vol. 46, no. 1, pp. 131-150, 1999.

[9] D. Motamedi and S. Mohammadi, "Dynamic analysis of fixed cracks in composites by the extended finite element method," Eng. Fract. Mech., vol. 77, no. 17, pp. 3373-3393, 2010.

[10] I. Nistor, O. Pantalé, and S. Caperaa, "Numerical implementation of the extended finite element method for dynamic crack analysis," Adv. Eng. Softw., vol. 39, no. 7, pp. 573-587, 2008.

[11] O. A. Pekau and C. Yuzhu, "Failure analysis of fractured dams during earthquakes by DEM," Eng. Struct., vol. 26, no. 10, pp. 1483-1502, 2004.

[12] F. Javanmardi, P. Léger, and R. Tinawi, "Seismic structural stability of concrete gravity dams considering transient uplift pressures in cracks," Eng. Struct., vol. 27, no. 4, pp. 616-628, 2005.

[13] J. Wang, G. Lin, and J. Liu, "Static and dynamic stability analysis using 3D-DDA with incision body scheme," Earthq. Eng. Eng. Vib., vol. 5, no. 2, pp. 273-283, 2006.

[14] O. A. Pekau and X. Zhu, "Three-degree-of-freedom rigid model for seismic analysis of cracked concrete gravity dams," J. Eng. Mech., vol. 132, no. 9, pp. 979-989, 2006.

[15] X. Zhu and O. A. Pekau, "Seismic behavior of concrete gravity dams with penetrated cracks and equivalent impact damping,” Eng. Struct., vol. 29, no. 3, pp. 336-345, 2007.

[16] M. Mirzayee, N. Khaji, and M. T. Ahmadi, "A hybrid distinct element-boundary element approach for seismic analysis of cracked concrete gravity dam-reservoir systems," Soil Dyn. Earthq. Eng., vol. 31, no. 10, pp. 1347-1356, 2011.

[17] J. M. Melenk and I. Babuška, "The partition of unity finite element method: basic theory and applications," Comput. Methods Appl. Mech. Eng., vol. 139, no. 1-4, pp. 289-314, 1996.

[18] S. Abaqus, "6.11. ABAQUS," CAE User's Man., 2011.

[19] S. Zhang, G. Wang, and X. Yu, "Seismic cracking analysis of concrete gravity dams with initial cracks using the extended finite element method," Eng. Struct., vol. 56, pp. 528-543, 2013.

[20] D. C. Simulia and S. ABAQUS, "6.11 analysis user's manual," Provid. RI, 2011.

[21] N. Sukumar, Z. Y. Huang, J. Prévost, and Z. Suo, "Partition of unity enrichment for bimaterial interface cracks," Int. J. Numer. Methods Eng., vol. 59, no. 8, pp. 1075-1102, 2004.

[22] N. Sukumar and J.-H. Prévost, "Modeling quasi-static crack growth with the extended finite element method Part I: Computer implementation," Int. J. Solids Struct., vol. 40, no. 26, pp. 7513-7537, 2003.

[23] T. Elguedj, A. Gravouil, and A. Combescure, "Appropriate extended functions for X-FEM simulation of plastic fracture mechanics," Comput. Methods Appl. Mech. Eng., vol. 195, no. 7-8, pp. 501-515, 2006.

[24] S. Mridha and D. Maity, "Experimental investigation on nonlinear dynamic response of concrete gravity dam-reservoir system," Eng. Struct., vol. 80, pp. 289-297, 2014. 
[25] H. Mohammadnezhad, M. Ghaemian, and A. Noorzad, "Seismic analysis of dam-foundation-reservoir system including the effects of foundation mass and radiation damping," Earthq. Eng. Eng. Vib., vol. 18, no. 1, pp. 203-218, 2019, doi: 10.1007/s11803-019-0499-4.
[26] A. Pirooznia, "Seismic Improvement of Gravity Dams Using Isolation Layer in Contact Area of Dam-Reservoir in Smeared Crack Approach," Iran. J. Sci. Technol. - Trans. Civ. Eng., vol. 43, no. 2, pp. 137-155, 2019, doi: 10.1007/s40996-018-01116. 